\title{
Quantum Theory of Particles with Spin Zero and One Half in External Fields $\star$
}

\author{
R. SEILER \\ Department of Physics, University of Pittsburgh, Pittsburgh, Pennsylvania, USA
}

Received October 18, 1971

\begin{abstract}
The unitary (pseudo unitary) time-evolution operator for a particle with spin half (zero) in an external time-dependent electromagnetic (scalar) field is used to generate a Bogoliubov automorphism on the algebra of the free in field. For the case of an electric external field (scalar field) a finite expression for $\Omega_{\text {out }}$ is given and the $S$-matrix constructed. The latter is unitary and implements the Bogoliubov automorphism. Theorems by Shale and Stinespring are rederived.
\end{abstract}

\section{Introduction}

Numerous papers have been devoted to the subject of quantum theory of particles with spin zero and one half in external fields. The formal aspects were well developed twenty years ago in particular through the work of Feynman [1], Salam and Mathews [2], and Schwinger [3]. A mathematical treatment of the theory is the purpose of this article.

Several authors have prepared ground for such an attempt. Capri [4] explains lucidly the "reduction to a $c$-number problem" (Section 4 and 5), Boongarts [5] treats at length the case of a spin $1 / 2$ oarticle in a stationary external electromagnetic field. Verifying the assumptions of a theorem by Shale and Stinespring [6] he proves existence of a time evolution operator in Fock space under conditions essentially the same as the ones in Theorem 6 for the case of time dependent external fields. In a previous paper [7] the existence of an out vacuum in the Fock space of the infield is discussed and assumptions necessary for the out-vacuum to exist are verified for the first model to be analyzed below (Section 4).

In the second and the third section the classical theory of a spin zero and spin $1 / 2$ particle in an external field is reviewed to the extent necessary for quantization. Specifically we consider two models, the

* Supported in part by the U.S. Atomic Energy Commission under Contract No. AT-30-1-3829. 
first one being characterized by the Cauchy problem

$$
\begin{aligned}
\left(\square+m^{2}\right) \phi(x) & =V(x) \phi(x) \\
V(t, \boldsymbol{x}) & =0 \text { for }|t|>T \\
\phi(t, \boldsymbol{x}) & \phi_{\text {in }}(t, \boldsymbol{x}) \text { for } t<-T,
\end{aligned}
$$

the second one by

$$
\begin{aligned}
(-i \not \partial+m) \psi(x) & =A(x) \psi(x) \\
A_{\mu}(t, \boldsymbol{x}) & =0 \text { for }|t|>T \\
\psi(t, \boldsymbol{x}) & =\psi_{\text {in }}(t, \boldsymbol{x}) \text { for } t<-T .
\end{aligned}
$$

Pseudounitarity is proved for the time evolution operator of the spin zero particle (Theorem 1). The $c$-number time evolution operators are then used to construct automorphisms of the free field algebras (reduction to $c$-number problem). They solve the $q$-number Cauchy problem (Theorem 2 for the first, Theorem 5 for the second model) and preserve the canonical structure (Bogoliubov automorphisms) due to pseudounitarity respectively unitarity of the classical $S$-matrix. For both models an explicit form of the out-vacuum is given (Theorem 3 , respectively Theorem 6) under provisions having been established previously for the first model. They are shown to be satisfied for the second model in case of an external electric field only, $\boldsymbol{A}=0$ (Theorem 7). A peculiarity of the fermion field has to be coped with, already familiar from the case of finite degrees of freedom (remark preceding Theorem 6). The vacua are then used to construct the $S$-matrix which is unitary. This is explicitly shown for the first model. The argument depends crucially upon pseudounitarity (not just pseudoisometry) of the classical $S$-matrix. Existence of a unitary $S$-matrix for both models could have been deduced directly from Theorem 4 respectively Theorem 7 and results of Shale (spin zero) respectively Shale and Stinespring [6] (spin one half). The latter in the formulation of Araki [8] is compared with Theorem 6 (Section 5).

Finally we remark that the discussion of the first model could probably be shortened considerably making use of results by Kristensen, Mejlbo and Poulsen [9].

\section{Classical Theory for Spin Zero Particles}

In order to discuss the Cauchy problem for a classical system it is useful to rewrite (1.1) in terms of particle-antiparticle amplitudes $a$ and $b$. Simultaneously, we introduce some notations. The amplitudes $a$ and $b$ are linked to the $c$-number field $\phi$ and it's conjugate momentum $\pi$ in the 
well known manner,

$$
\begin{aligned}
& a=\left(\frac{\omega}{2}\right)^{1 / 2} \phi+i\left(\frac{1}{2 \omega}\right)^{1 / 2} \pi \\
& \bar{b}=\left(\frac{\omega}{2}\right)^{1 / 2} \phi-i\left(\frac{1}{2 \omega}\right)^{1 / 2} \pi
\end{aligned}
$$

where - denotes complex conjugation and $\omega$ is the frequency operator, $\omega=\left(-\Delta+m^{2}\right)^{1 / 2}$. The symbol + will be used for the adjoint of operators. The Cauchy problem (1.1) can be rewritten in the form of a Schrödinger equation

$$
\begin{aligned}
i \partial_{t} \phi & =H \phi, \\
\phi(t, x) & =\phi_{\text {in }}(t, x) \text { for } t \leqq-T
\end{aligned}
$$

$\phi$ is a two component function $\phi(x)=\left(\begin{array}{l}a(x) \\ b(x)\end{array}\right)$ and $H$ a $2 \times 2$ matrix operator

$$
\begin{aligned}
H & =H_{0}+H_{1} \\
H_{0} & =\left(\begin{array}{rr}
\omega & \\
& -\omega
\end{array}\right), \quad H_{1}=\left(\begin{array}{rr}
-v & -v \\
v & v
\end{array}\right), \quad v=\left(\frac{1}{2 \omega}\right)^{1 / 2} V\left(\frac{1}{2 \omega}\right)^{1 / 2}
\end{aligned}
$$

The Hilbert space $\mathfrak{H}$ we are dealing with is the direct sum of two spaces of square integrable functions $L^{2}$

$$
\mathfrak{H}=L^{2}\left(d^{3} x\right) \oplus L^{2}\left(d^{3} x\right) .
$$

The domain $D\left(H_{0}\right)$ of the selfadjoint operator $H_{0}$ is most conveniently given in the space of Fourier transformed functions $\hat{\mathfrak{H}}$

$$
D\left(H_{0}\right)=L^{2}\left(d^{3} p\left(p^{2}+m^{2}\right)\right) \oplus L^{2}\left(d^{3} p\left(p^{2}+m^{2}\right)\right) .
$$

The physical charge of a field can be used to define an indefinite scalar product in $\mathfrak{H}$

$$
[\phi, \phi]=i \int d^{3} x \phi(x) \overleftrightarrow{\partial_{0}} \phi(x)
$$

Introducing the metric tensor $g$

$$
g=\left(\begin{array}{ll}
i & \\
& -i
\end{array}\right)
$$

it can be written in terms of the positive definite scalar product

$$
[\phi, \phi]=(\phi, g \phi)
$$


$H$ is pseudo-selfadjoint with respect to $g$

$$
g H^{+} g=H \text {. }
$$

The following theorem summarizes results which will be important in the sequel

Theorem 1. Let $V$ be a testfunction in $\subseteq$ and $V(t, \cdot)=0$ for $|t|>T$. Then there exists a time evolution operator $U\left(t_{2}, t_{1}\right)$, norm continuous in $t_{1} \in R$ and $t_{2} \in R$, such that

i) $U\left(t_{2}, t_{1}\right) U\left(t_{1}, t_{0}\right)=U\left(t_{2}, t_{0}\right), U(t, t)=1, t_{2}>t_{1}>t_{0}$.

ii) $\phi(t)=U\left(t, t_{0}\right) \phi\left(t_{0}\right), \phi\left(t_{0}\right) \in D\left(H_{0}\right)$ is a solution of the Cauchy problem (1.1) with data $\phi\left(t_{0}\right)$.

iii) $U\left(t_{0}, t_{1}\right)$ is pseudo-unitary with respect to the physical metric $g$, $U\left(t_{0} t_{1}\right) g U^{+}\left(t_{0} t_{1}\right)=U^{+}\left(t_{0}, t_{1}\right) g U\left(t_{0}, t_{1}\right)=g$.

iv) $U$ induces a causal propagation, i.e. if $\phi\left(t_{0}\right)$ has support in the complement of $\{x|| x \mid \leqq R\}$ then $\phi(t), t \geqq t_{0}$, has support in the complement of $\left\{\boldsymbol{x}|| \boldsymbol{x}|+| t-t_{0} \mid \leqq R\right\}$.

All but statement iii) are standard results [10]. We will now give a proof for iii) which at the same time reproduces i) and ii) using the concept of the product integral [11]. Since $H_{0}$ is selfadjoint it is appropriate to investigate the Cauchy problem (2.2) in the interaction picture

$$
\begin{gathered}
i \partial_{t} \tilde{\phi}=\tilde{H}_{1}(t) \tilde{\phi} \\
\tilde{\phi}(t, \boldsymbol{x})=\phi_{\mathrm{in}}(0, \boldsymbol{x}) \quad \text { for } t \leqq-T \\
\tilde{\phi}(x)=e^{i H_{0} t} \phi(x)=\left(\begin{array}{l}
a_{t}(\boldsymbol{x}) \\
\bar{b}_{t}(\boldsymbol{x})
\end{array}\right)=\left(\begin{array}{l}
e^{i \omega t} a(x) \\
e^{-i \omega t} \bar{b}(x)
\end{array}\right) \\
\tilde{H}_{1}(t)=g^{i H_{0} t} H_{1} e^{-i H_{0} t} .
\end{gathered}
$$

We first show the following statement leading immediately to a proof of Theorem 1

Lemma 1. Under the assumptions of Theorem 1 there exists a time evolution operator $\tilde{U}\left(t_{1}, t_{2}\right)$ such that

i) $\tilde{U}\left(t_{2} t_{1}\right) \tilde{U}\left(t_{1}, t_{0}\right)=\tilde{U}\left(t_{2}, t_{0}\right), \tilde{U}(t, t)=1, t_{2} \geqq t_{1} \geqq t_{0}$.

ii) $\tilde{\phi}(t)=\tilde{U}(t,-T) \phi_{\text {in }}(0, x)$ is a solution of the Cauchy problem (2.9).

iii) $\tilde{U}$ is pseudo-unitary.

Proof. Existence of $\tilde{U}\left(t_{1}, t_{2}\right)$ is guaranteed [12] by the statement of the following

Lemma 2. Under the assumption of Theorem $1 H_{1}(t)$ is a bounded operator in $\mathfrak{S}$ and norm continuous in $t$. 
Proof. 1. $H_{1}(t)$ is bounded: It is sufficient to prove that $H_{1}$ is bounded. However, each term $v$ in the matrix operator $H_{1}$ is a bounded operator on $L^{2}\left(d^{3} p\right)$ from which the assertion follows.

2. $\left[\mathrm{H}_{0}, H_{1}\right]$ is bounded: It is sufficient to prove that $\omega^{-1 / 2} V \omega^{1 / 2}$ is a bounded operator on $L^{2}\left(d^{3} p\right)$. Consider the expression

$$
\left(f, \omega^{-1 / 2} V \omega^{1 / 2} g\right)=\int d^{3} p d^{3} q \bar{f}(p) F(p, q) V(p-q) g(q)
$$

where we denoted by $F$ the function

$$
F(p, q)=\left(p^{2}+m^{2}\right)^{-1 / 4}\left(q^{2}+m^{2}\right)^{1 / 4} .
$$

The right hand side of (2.10) can be estimated in the following manner

However since

$$
\begin{aligned}
\left|\int d^{3} p d^{3} q \overline{f(p)} F(p, q) V(p-q) g(q)\right| \\
\quad \leqq\left(\int d^{3} s|V(s)| \sup _{p}|F(p, p-s)|\right)\|f\|\|g\| .
\end{aligned}
$$

we get

$$
\sup _{p}|F(p, p-s)| \leqq 4 m^{2}+s^{2}
$$

$$
\left|\left(f, \omega^{-1 / 2} V \omega^{1 / 2} g\right)\right| \leqq \text { const }\|f\|\|g\| .
$$

Combining the last inequality with

$$
\left\|\omega^{-1 / 2} V \omega^{1 / 2}\right\|=\sup _{\|f\|=\|g\|=1}\left|\left(f, \omega^{-1 / 2} V \omega^{1 / 2} g\right)\right|
$$

the statement follows.

3. $\tilde{H}_{1}(t)$ is norm continuous: Let $\phi$ be an element in $D\left(H_{0}\right) \cdot H(t) \phi$ is strongly differentiable

$$
\frac{d}{d t} \tilde{H}_{1}(t) \phi=i e^{i H_{0} t}\left[H_{0}, H_{1}\right] e^{-i H_{0} t} \phi
$$

$$
+(\text { operator uniformly bounded in } t) \phi \text {. }
$$

Integrating this equation we get

$$
\begin{aligned}
\tilde{H}_{1}\left(t_{2}\right) \phi-\tilde{H}_{1}\left(t_{1}\right) \phi= & i \int_{t_{1}}^{t_{2}} d t e^{i H_{0} t}\left[H_{0}, H_{1}\right] e^{-i H_{0} t} \phi \\
& + \text { (operator uniformly bounded in } t) \phi .
\end{aligned}
$$

Having just proved that $\left[H_{0}, H_{1}\right]$ is bounded we can estimate the right hand side of (2.15) as follows

$$
\left\|\int_{t_{1}}^{t_{2}} d t e^{i H_{0} t}\left[H_{0}, H_{1}\right] e^{-i H_{0} t} \phi\right\| \leqq\left(t_{2}-t_{1}\right) \text { const }\|\phi\| .
$$


Since $D\left(H_{0}\right)$ is dense in $\mathfrak{H}$ the last two relations imply the statement made in Lemma 2

$$
\left\|\hat{H}\left(t_{2}\right)-\hat{H}\left(t_{1}\right)\right\| \leqq\left|t_{2}-t_{1}\right| \text { const } .
$$

In continuation of the proof of Lemma 1 we define the product integral over $\tilde{H}_{1}(t)$ which exists as limit in the norm topology due to Lemma 2 as we mentioned earlier:

$$
\begin{aligned}
\tilde{U}\left(t_{2}, t_{1}\right) & =\prod_{t_{1}}^{t_{2}}\left(1-i \tilde{H}_{1}(t) d t\right)=\lim _{N \rightarrow \infty} \tilde{U}_{N}\left(t_{2}, t_{1}\right), \\
\tilde{U}_{N}\left(t_{2}, t_{1}\right) & =e^{-i \Delta t \tilde{H}_{1}\left(t_{2}\right)} \ldots e^{-i \Delta t \tilde{H}_{1}\left(t_{1}+\Delta t\right)} \\
\Delta t & =\frac{1}{N}\left(t_{2}-t_{1}\right) .
\end{aligned}
$$

Defining the bounded operators

$$
U\left(t_{2}, t_{1}\right)=e^{-i H_{0} t_{2}} \tilde{U}\left(t_{2}, t_{1}\right) e^{i H_{0} t_{1}}
$$

one readily checks the first two statements of Theorem 1 .

Turning to the last statement of Lemma 1, we notice the following: The same way as $\tilde{U}$ has been defined as the norm limit of $\tilde{U}_{N}$ the adjoint $\tilde{U}^{+}$is the norm limit of

$$
\tilde{U}_{N}^{+}=e^{i \Delta t \tilde{H}_{1}\left(t_{1}+\Delta t\right)} \ldots e^{i \Delta t \tilde{H}_{1}\left(t_{2}\right)} .
$$

Since

$$
\tilde{U}_{N}^{+} g \tilde{U}_{N}=g=\tilde{U}_{N} g \tilde{U}_{N}^{+}
$$

as a consequence of the definitions (2.15) and (2.18) and because of the norm continuity of the product operation for bounded operators on a Hilbert space $\tilde{U}$ is shown to be pseudo-unitary

$$
\tilde{U}^{+} g \tilde{U}=\tilde{U} g \tilde{U}^{+}=g .
$$

However, this proves the third statement of Theorem 1 too, due to (2.20).

Concluding this section we should like to draw attention to two facts: First, the classical $S$-matrix $S_{\mathrm{cl}}$ mapping free incoming states for time $t=0$ onto free outgoing states at the same time is pseudo-unitary,

$$
\tilde{\phi}_{\text {out }}=S_{\text {cl }} \tilde{\phi}_{\text {in }} .
$$

This is just a special case of the pseudo-unitarity of $\tilde{U}$ because of

$$
S_{\mathrm{cl}}=\tilde{U}(T,-T) \text {. }
$$


Secondly, one could replace the product integral in (2.18) by a Dyson integral

$$
\tilde{U}\left(t_{2}, t_{1}\right)=T \exp -i \int_{t_{1}}^{t_{2}} d t \tilde{H}_{1}(t)
$$

defined as a power series and converging in norm due to Lemma 2.

\section{Classical Theory for Spin 1/2 Particles}

In analogy to the previous case we rephrase the classical Cauchy problem (1.2) in terms of particle-antiparticle amplitudes $y_{t}(x, s), b_{t}(x, s)$, (interaction-picture). Let $\psi(x)$ be a solution of (1.2) in the Hilbert space $\mathfrak{H}_{1 / 2}$

$$
\begin{aligned}
\mathfrak{S}_{1 / 2} & =L^{2}\left(d^{3} x\right) \otimes C^{4} \\
(\psi, \psi) & =\int d^{3} x \bar{\psi}(x) \psi(x) .
\end{aligned}
$$

Traditionally, $\psi$ is decomposed into plane waves [13] (tacitly assuming summation over $s, s=1,2$ )

$$
\begin{aligned}
\psi(x)= & \left(\frac{1}{2 \pi}\right)^{3 / 2} \int d^{3} p\left(\frac{m}{\omega(p)}\right)^{1 / 2} \\
& \cdot\left(e^{-i p x} u(\boldsymbol{p}, s) a_{t}(\boldsymbol{p}, s)+e^{i p x} v(\boldsymbol{p}, s) b_{t}^{+}(p, s)\right) .
\end{aligned}
$$

The vector $\hat{\phi}$

is an element of $\hat{\mathfrak{Y}}_{1 / 2}$

$$
\hat{\phi}(\boldsymbol{p})=\left(\begin{array}{l}
a_{t}(\boldsymbol{p}, \cdot) \\
b_{t}(\boldsymbol{p}, \cdot)
\end{array}\right)
$$

$$
(\hat{\phi}, \hat{\phi})=\sum_{s} \int d^{3} p\left(\left|a_{t}(\boldsymbol{p}, s)\right|^{2}+\left|\bar{b}_{t}(\boldsymbol{p}, s)\right|^{2}\right)=(\psi, \psi) .
$$

$\psi(x)$ can be rewritten in terms of the bounded operator

in $\mathfrak{S}_{1 / 2}$

$$
\left(\frac{m}{\omega}\right)^{1 / 2}(u(-i \hat{\partial}, s), v(i \hat{\partial}, s))
$$

$$
\psi(x)=\left(\frac{m}{\omega}\right)^{1 / 2}\left(u(-i \partial, s) a_{t}(x, s)+v(i \hat{\partial}, s) \bar{b}_{t}(x, s)\right)
$$

where we used the abbreviations

$$
\left(\begin{array}{l}
a_{t}(x, s) \\
b_{t}(x, s)
\end{array}\right)=\left(\frac{1}{2 \pi}\right)^{3 / 2} \int d^{3} p e^{-i p x}\left(\begin{array}{l}
a_{t}(\boldsymbol{p}, s) \\
b_{t}(\boldsymbol{p}, s)
\end{array}\right) .
$$


Eq. (3.5) can be inverted using well known orthogonality relations among the plane wave solution of the Dirac equation [13]

$$
\phi_{t}(x)=\left(\begin{array}{l}
a_{t}(x) \\
b_{t}^{+}(x)
\end{array}\right)=\left(\begin{array}{l}
\left(\frac{m}{\omega}\right)^{1 / 2} \bar{u}(-i \partial, s) \psi(x) \\
\left(\frac{m}{\omega}\right)^{1 / 2} \bar{v}(i \partial, s) \psi(x)
\end{array}\right) .
$$

The Cauchy problem (1.2) in terms of particle antiparticle amplitudes and the interaction picture has the form

$$
\begin{aligned}
& i \partial_{t} \tilde{\phi}=\tilde{H}_{1}(t) \tilde{\phi} ; \quad \tilde{\phi}(t)=\phi_{\text {in }}(0, \boldsymbol{x}), t<-T \\
& \tilde{H}_{1}(t)=e^{i H_{0} t} H_{1} e^{-i H_{0} t} \quad \tilde{\phi}=e^{i H_{0} t} \phi=\left(\begin{array}{l}
a_{t}(\boldsymbol{x}, s) \\
\bar{b}_{t}(\boldsymbol{x}, s)
\end{array}\right) \\
& H_{0}=\left(\begin{array}{cc}
\omega & 0 \\
0 & -\omega
\end{array}\right) \\
& H_{1}=\left(\frac{m}{\omega}\right)^{1 / 2}\left(\begin{array}{ll}
\tilde{u}(-i \partial, s) A u\left(-i \partial, s^{\prime}\right) & \tilde{u}(-i \partial, s) A v\left(i \partial, s^{\prime}\right) \\
\tilde{v}(i \partial, s) A u\left(-i \partial, s^{\prime}\right) & \tilde{v}(i \partial, s) A v\left(i \partial, s^{\prime}\right)
\end{array}\right)\left(\frac{m}{\omega}\right)^{1 / 2}
\end{aligned}
$$

Notice the close analogy to the previous case (2.9).

We will refrain from proving anything concerning the solution of the Cauchy problem (1.2) and (3.8) since it would parallel to closely the reasoning in the previous case (Lemma 1). Let us however, state the following result:

Lemma 3. Let $A_{\mu}(x)$ be a test function in $\subseteq\left(R^{4}\right)$ and $A_{\mu}(t, x)=0$ for $|t|>T$. Then there exists a time evolution operator $\tilde{U}\left(t_{1}, t_{2}\right)$ such that

i) $\tilde{U}\left(t_{2}, t_{1}\right) \tilde{U}\left(t_{1}, t_{0}\right)=\tilde{U}\left(t_{2}, t_{0}\right), \tilde{U}\left(t_{1} t\right)=1, t_{2} \geqq t_{1} \geqq t_{0}$.

ii) $\tilde{\phi}(t)=\tilde{U}\left(t_{1}-T\right) \phi_{\mathrm{in}}(0)$ is a solution of the Cauchy problem (3.8).

iii) $\tilde{U}$ is unitary.

The above statement implies through (2.20) again a theorem analogous to Theorem 1. Notice however, that pseudo-unitarity has been replaced by unitarity due to the positive definite charge in a $c$ number theory for particles with half integer spin. The $S$-matrix is defined as previously (2.25). Again the norm continuity of $\tilde{H}_{1}(t)$ can be shown and allows $\tilde{U}$ to be written in terms of a product integral or a norm convergent Dyson integral.

\section{Quantum Theory for Spin Zero Particles}

The $c$-number solution of the Cauchy problem (1.2) can be used to generate a time evolution automorphisme $\alpha_{t}, t \in R$, on the algebra $\mathfrak{B}(\mathfrak{F})$ of the free in field thereby generating the solution of the $q$-number 
Cauchy problem (reduction to $c$-number problem). Some properties of $\alpha$ are compiled in

Theorem 2. Provided the statements in Theorem 1 hold the mapping $\alpha_{t}$.

$$
\begin{aligned}
\left(\alpha_{t} \phi_{\mathrm{in}}\right)(\boldsymbol{x}) & =\left(\begin{array}{l}
a_{t}(\boldsymbol{x}) \\
b_{t}^{+}(\boldsymbol{x})
\end{array}\right):=\tilde{U}(t) \phi_{\mathrm{in}}(\boldsymbol{x}) \\
\tilde{\phi}_{\mathrm{in}}(\boldsymbol{x}) & :=\tilde{\phi}(0, \boldsymbol{x}) \\
\tilde{U}(t) & :=\tilde{U}(t,-T)
\end{aligned}
$$

generates an automorphisme of the algebra of bounded operators $\mathfrak{B}(\mathfrak{F})$ in the Fock space $\mathfrak{i}$ of the field at $t=0$. In particular

$$
\phi_{\text {out }}(\boldsymbol{x})=S_{\mathrm{cl}} \tilde{\phi}_{\text {in }}(\boldsymbol{x}) \text {, }
$$

$\alpha_{t}$ has the following properties,

i) $\left(\alpha_{t} \phi_{\text {in }}\right)(x)$ restricted to the linear subspace $\mathfrak{D}_{\text {in }}$ of states with smooth wave functions and only finitely many particles

$$
\mathfrak{D}_{\mathrm{in}}=\left\{\left\langle\prod_{m=1}^{M} \prod_{n=1}^{N} a_{\mathrm{in}}^{+}\left(f_{i}\right) b_{\mathrm{in}}^{+}\left(g_{i}\right) \Omega_{\mathrm{in}}\right\rangle \mid f_{i}, g_{i} \text { test functions; } M, N \in Z_{+}\right\}
$$

is the unique solution of the q-number Cauchy problem (2.9).

ii) $\alpha_{t}$ is local; i.e. the field $\phi(x)$ defined in terms of the particle operators $a$ and $b$ in $\phi(x)(2.1)$

$$
\phi(x)=\left(\alpha_{t} \phi_{\text {in }}\right)(x)=e^{-i H_{0} t} \tilde{U}(t) \phi_{\text {in }}(x)
$$

is local,

$$
\left[\phi(x), \phi\left(x^{r}\right)\right]=0 \quad \text { if } \quad\left(x-x^{r}\right)^{2}<0 .
$$

iii) $\alpha_{t}$ is a Bogoliubov automorphisme (preserves canonical structure). Preceding to the proof of Theorem 2 we wish to make two remarks:

i) It is well known that $\mathfrak{B}(\mathfrak{F})$ has the two alternative representations [14]

$$
\begin{aligned}
\mathfrak{B}(\mathfrak{F})= & \left\{e^{i\left(a_{\text {in }}(f)+a_{\text {in }}^{+}(f)\right)}, e^{a_{\text {in }}(g)-a_{\text {in }}^{+}(g)}, e^{i\left(b_{\text {in }}(f)+b_{\text {1n }}^{+}(f)\right)},\right. \\
& \left.e^{b_{\text {in }}(g)-b_{\text {i }}^{+}(g)} \mid f, g \text { real test functions }\right\}^{\prime \prime} \\
= & \left\{\ldots f \in L^{2}, g \in L^{2} \text { real }\right\}^{\prime \prime}
\end{aligned}
$$

where" denotes the double commutand.

(ii) Under the provision of statement i) in Theorem 2, $\phi(x)(4.4)$ is the solution of the $q$-number Cauchy problem (2.2) on $\mathfrak{D}_{\text {in }}$.

Proof of Theorem 2. 1. $\alpha_{t}$ is an automorphisme of $\mathfrak{B}(\mathfrak{F})$ : According to the corollary of Theorem $1, \tilde{U}(t)$ maps $\mathfrak{H}$ onto $\mathfrak{H}$. Together with (4.5) this shows that $\alpha_{t}$ is an automorphisme.

10 Commun math Phys, Vol. 25 
2. $\alpha_{t} \phi_{\text {in }}$ is a solution of the $q$-number Cauchy problem: For any $F \in \mathfrak{H}$ and fixed $x \in \mathfrak{D}_{\text {in }}$

$$
F \rightarrow \phi_{\text {in }}(F) x=\int d^{3} y F(y) \phi_{\text {in }}(y) x
$$

is continuous [15]. Therefore, the second statement of Theorem 1 implies

$$
\begin{aligned}
i \partial_{t}\left(\alpha_{t} \phi_{\mathrm{in}}\right)(F) x & =\phi_{\mathrm{in}}\left(i \partial_{t} \tilde{U}^{T}(t) F\right) x \\
& =\phi_{\mathrm{in}}\left(\tilde{H}_{1}^{T} \tilde{U}^{T}(t) F\right) x
\end{aligned}
$$

which proves the assertion up to the verification of the initial data, which however, is trivial.

3. $\alpha_{t} \phi_{\text {in }}$ is the unique solution of the $q$-number problem: Let $x$ and $F$ be defined as above and let $\psi_{t}$ be a solution of the Cauchy problem on $\mathfrak{D}_{\text {in }}$. Then the differential equation for $\alpha_{t} \phi_{\text {in }}$ and $\psi_{t}$ implies

$$
\left(\alpha_{t} \phi_{\text {in }}-\psi_{t}\right) \mathrm{x}=\text { const } x \forall t \in R \text {. }
$$

However, the initial condition force the constant to vanish.

4. $\alpha_{t}$ is local: It is not difficult to convince ones self that the commutator $\left[\phi(x), \phi^{+}\left(x^{\prime}\right)\right]$ is a $c$-number. Therefore

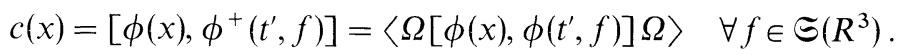

But $c(x)$ is a solution of the differential Eq. (1.2) hence a smooth function in $t$ and vanishes for $t=t^{\prime}$ and $\boldsymbol{x}$ outside the support of $f$. By Hugghens principle for the $c$-number solutions of $(1.2)[10] c(x)$ has to vanish for $x$ space-like to the set $\left\{\left(t^{\prime}, \boldsymbol{x}^{\prime}\right) \mid \boldsymbol{x}^{\prime} \in \operatorname{supp} f\right\}$.

5. $\alpha_{t}$ is a Bogoliubov automorphism: We are going to show that due to the pseudo-unitarity of $\tilde{U}$ (corollary to Theorem 1) $a_{t}(\boldsymbol{x})$ and $b_{t}(\boldsymbol{x})$ are canonical,

$$
\begin{aligned}
& {\left[y_{t}(f), a_{t}^{+}(g)\right]=(f, g),} \\
& {\left[b_{t}(f), b_{t}^{+}(g)\right]=(f, g)}
\end{aligned}
$$

and all other commutators will vanish. Due to (4.1) the first commutator is given by (we suppress the arguments in $\tilde{U}(t,-T)$ )

$$
\begin{gathered}
{\left[a_{\mathrm{in}}\left(\tilde{U}_{11}^{+} f\right)+b_{\mathrm{in}}^{+}\left(\tilde{U}_{12}^{+} f\right), a_{\mathrm{in}}^{+}\left(\tilde{U}_{11}^{+} g\right)+b_{\mathrm{in}}\left(\tilde{U}_{12}^{+} g\right)\right]=\left(f,\left(\tilde{U}_{11} \tilde{U}_{11}^{+}-\tilde{U}_{12} \tilde{U}_{12}^{+}\right) g\right)} \\
=(f, g) .
\end{gathered}
$$

For the last step we used (2.23) and the canonical structure of the free in field $\phi_{\text {in }}$. All the other commutators can be computed along the same lines.

Next we have to discuss whether the automorphism $\alpha$ of the Fock space algebra $\mathfrak{B}$ can be unitarily implemented. A necessary and as it 
can be shown also sufficient condition for the existence of a unitary operator $\mathfrak{U}(t), t \in R$, such that

$$
\alpha_{t} \phi_{\mathrm{in}}=\mathfrak{U}(t) \phi_{\mathrm{in}} \mathfrak{U}^{-1}(t)=\left(\begin{array}{l}
a_{t} \\
b_{t}^{+}
\end{array}\right)
$$

is given in Theorem 3. The proof will be constructive and therefore of some practical interest.

Theorem 3. If $\tilde{U}(t)$ is pseudo-unitary the operator

$$
L(t):=\tilde{U}_{11}^{-1}(t) \tilde{U}_{12}(t)
$$

is well defined. In particular if L(t) is Hilbert-Schmidt (H.S.),

$$
\begin{aligned}
& L(t)=\sum_{i=1}^{\infty} \lambda_{i} f_{i} \otimes g_{i}, \quad \lambda_{i} \geqq 0, \quad \sum_{i=1}^{\infty} \lambda_{i}^{2}<\infty \\
& \left\{f_{i}\right\},\left\{g_{i}\right\} \text { each orthogonal basis of } L_{2},
\end{aligned}
$$

the nontrivial vector $\chi$,

$$
\chi=\exp -\sum_{i} \lambda_{i} a_{i}^{+} b_{i}^{+} \Omega_{\mathrm{in}}, \quad y_{i}=a_{\mathrm{in}}\left(f_{i}\right), \quad b_{i}=b_{\mathrm{in}}\left(\bar{g}_{i}\right)
$$

is up to normalization and a phase the unique vacuum $\Omega_{t}$ for $\alpha_{t} \phi_{\text {in }}$. The time evolution operator $\mathfrak{U}(t)$ acting in $\mathfrak{F}$ and in particular the $S$-matrix

is unitary.

$$
\subseteq=\mathfrak{U}(T)
$$

Remark: If $L(t)$ is H.S. the operator

$$
M(t)=-\left(S_{11}^{+}\right)^{-1} S_{21}^{+}
$$

is H.S. too because the class of H.S. operators is a + -invariant ideal in the set of bounded operators $\mathfrak{B}\left(L^{2}\right)$ in $L^{2}$.

Proof. 1. The operator $L(t)$ is well defined: According to Theorem 1 $\tilde{U}$ is pseudo-unitary (7.23), in particular

$$
\tilde{U}_{11}^{+} \tilde{U}_{11}=1+\tilde{U}_{12}^{+} \tilde{U}_{12} .
$$

Hence

$$
\tilde{U}_{11} \tilde{U}_{11} \geqq 1
$$

which forces the kernel of $\tilde{U}_{11}$ to vanish. $\tilde{U}_{11}$ is therefore invertible and the inverse $\tilde{U}_{11}^{-1}$ is defined on the range $R\left(\tilde{U}_{11}\right)$ of $\tilde{U}_{11}$. The next argument shows that the range of $\tilde{U}_{11}$ is in fact the hole space $L^{2}$. Assume that $f$ is in the orthogonal complement of $R\left(\tilde{U}_{11}\right)$ :

$$
\left(f, \tilde{U}_{11} g\right)=0, \quad \forall g \in L^{2} .
$$


Hence $f$ is in the kernel of $\tilde{U}_{11}^{+}$. However, from pseudo-unitary one concludes

$$
\tilde{U}_{11} \tilde{U}_{12} \tilde{U}_{12}^{+}+1 \text {. }
$$

By the same argument as before $f$ has to vanish.

2. The $\lambda_{i}$ 's are strictly smaller than one: Again because of (4.16) it follows that

$$
\begin{aligned}
& 1=L L^{+}+\tilde{U}_{11}^{-1} \tilde{U}_{11}^{-1+} \\
& 1=\Sigma \lambda_{i}^{2} f_{i} \otimes f_{i}+\tilde{U}_{11}^{-1} \tilde{U}_{11}^{-1+}
\end{aligned}
$$

and this implies $\lambda_{i} \leqq 1$ : Let $\lambda_{0}=1$ and $f_{0}$ be the corresponding eigenvector of $L L^{+}$. Eq. (4.17) implies

$$
\tilde{U}_{11}^{-1} \tilde{U}_{11}^{-1+} f_{0}=0 \text {. }
$$

Neither $\tilde{U}_{11}^{-1}$ nor $\tilde{U}_{11}^{-1+}$ can have a nontrivial kernel because $\tilde{U}_{11}, \tilde{U}_{11}^{+}$ and their inverses are bounded and everywhere defined.

3. $\chi$ is a well defined vector in the Fock space of the in field: Consider the sequence $\left\{\chi_{N}\right\}$,

$$
\chi_{N}=\prod_{i=1}^{N} e^{-\lambda_{2} a_{i}^{+} b_{i}^{+}} \Omega_{\text {in }} .
$$

We claim $\left\{\chi_{N}\right\}$ is a Cauchy sequence. Let $M$ and $N$ be natural numbers. A straightforward calculation yields

$$
\left\|\chi_{N}-\chi_{M}\right\|^{2}=\left|\prod_{i=1}^{N} \frac{1}{1-\lambda_{i}^{2}}-\prod_{i=1}^{M} \frac{1}{1-\lambda_{i}^{2}}\right| .
$$

Hence $\left\{\chi_{N}\right\}$ is Cauchy if and only if the infinite product

$$
\prod_{i=1}^{\infty} \frac{1}{1-\lambda_{i}^{2}}, \quad \lambda_{i}<1
$$

is finite. However according to a lemma of $v$. Neumann [16] this is equivalent to $L$ being Hilbert-Schmidt, $\Sigma \lambda_{i}^{2}<\infty$.

Remark. A slight generalization shows that the sequence of operators

$$
\varrho_{N}=\prod_{i=1}^{Q} \prod_{j=1}^{P} a_{i}^{m_{J}} b_{j}^{n_{j}} \prod_{s=1}^{N} e^{-\lambda_{s} a_{s}^{+} b_{s}^{+}}
$$

$$
P, Q, m_{i}, n_{j} \text { positive integers }
$$

converges on the dense linear subspace $\mathfrak{E}_{\text {in }} \subset \mathfrak{F}$,

$$
\mathfrak{E}_{\mathrm{in}}=\left\{\left\langle\prod_{i=1}^{P} \prod_{j=1}^{Q} a_{i}^{+m_{\imath}} b_{j}^{+n_{j}} \Omega_{\mathrm{in}}\right\rangle \mid P, Q, m_{i}, n^{j} \text { positive integers }\right\} .
$$

4. $\chi$ is annihilated by $a_{t}$ and $b_{t}$

$$
a_{t}(x) \chi=b_{t}(x) \chi=0 .
$$


We check only the first one of the two equations. $a_{t}$ is given in terms of the in field as follows (4.1)

$$
a_{t}(f)=a_{\mathrm{in}}\left(\tilde{U}_{11}^{+} f\right)+b_{\mathrm{in}}^{+} \overline{\left(\tilde{U}_{12}^{+} f\right)} .
$$

Using (4.15) and (4.16) one proves that $\tilde{U}_{11}^{+}$is invertible and $\tilde{U}^{+-1}$ has range $R\left(\tilde{U}_{11}^{+-1}\right)=L^{2}$ the same way we proceeded to prove the analogous statement for $\tilde{U}$. Hence it is sufficient to show

$$
a_{t}\left(\tilde{U}_{11}^{+-1} f_{i}\right) \chi=0, \quad f_{i} \text { any element of the basis }\left\{f_{i}\right\} .
$$

Since the following relations hold on $\mathbb{E}_{\mathrm{in}}$

$$
a_{t}\left(\tilde{U}_{11}^{+-1} f_{i}\right)=a_{i}+\lambda_{i} b_{i}^{+}
$$

we are left with the verification of

$$
\left(a_{i}+\hat{i}_{\imath} b_{i}^{+}\right) \chi=0 .
$$

Due to (4.13) Eq. (4.22) reduces to the well known relation

$$
\left(a_{i}+\lambda_{i} b_{i}^{+}\right) e^{-\lambda_{\imath} a_{i}^{+} b_{i}^{+}} \Omega_{\text {in }}=0 .
$$

5. The time evolution operator $\mathfrak{U}(t)$ is unitary: Define the linear time evolution operator $\mathfrak{U}(t)$ on $\mathfrak{E}_{\text {in }}(4.13)$ as follows

$$
\begin{aligned}
& \mathfrak{U}(t) \Omega_{\text {in }}=\Omega_{t} \\
& \mathfrak{U}(t) y_{\text {in }}^{\#}=a_{t}^{\ddagger} \mathfrak{U}(t) \\
& \mathfrak{U}(t) b_{\text {in }}^{\sharp}=b_{t}^{\ddagger} \mathfrak{U}(t) .
\end{aligned}
$$

$a^{\#}$ stands for $a$ or $a^{+}$. It is readily seen from the canonical structure of $a_{\text {in }}, b_{\text {in }}$ as well as $a_{t}, b_{t}$ that $\mathfrak{U}$ is a densely defined isometry on $\mathfrak{F}$ :

$$
\mathfrak{U}^{+}(t) \mathfrak{U}(t)=1
$$

It remains to be shown that $\mathfrak{U}$ is actually unitary or equivalently, that the range $R(\mathfrak{U})$ of $\mathfrak{U}$ is dense in $\mathfrak{F}$.

Let us first show that there is an expression similar to (4.11) for $\Omega_{\text {in }}$ in terms of $a_{t}, b_{t}$ and $\Omega_{t}$. Recalling that under the assumption of the theorem $M$ (4.14),

$$
M=\Sigma \mu_{i} h_{i} \otimes k_{i}
$$

is H.S. We define on $\mathfrak{E}_{\text {in }}$

$$
\begin{aligned}
a_{i}^{\prime} & =a_{t}\left(k_{i}\right) \\
b_{i}^{\prime} & =b_{t}\left(\bar{k}_{i}\right) \\
\xi & =\exp -\sum_{i=1}^{\infty} \mu_{i} a_{i}^{\prime+} b_{i}^{\prime+} \Omega_{t}
\end{aligned}
$$


$\xi$ is well defined in $\mathfrak{F}$ the same way as $\chi$ (Section 4 and 5) and one shows that $\xi$ is annihilated by $y_{\mathrm{in}}, b_{\mathrm{in}}$. Hence the vector $\Omega_{\mathrm{in}}^{\prime}$

$$
\Omega_{\text {in }}^{\prime}=(\xi \xi)^{-1 / 2} \xi
$$

differs from $\Omega_{\text {in }}$ at most by a phase.

Denoting by $x$ an arbitrary element in $\mathfrak{F}$ there is in any $\varepsilon$-neighborhood of $x$ a second element $y$ which can be generated from $\Omega_{\text {in }}$ by a finite application of $y_{\text {in }}, b_{\text {in }}$

$$
\begin{aligned}
y= & \sum_{\substack{\text { finite } \\
\text { sum }}} c\left(m_{i}, n_{j}\right) a_{\mathrm{in}}^{+}\left(\tilde{U}_{11}^{-1} h_{1}\right)^{n_{1}} \ldots a_{\mathrm{in}}^{+}\left(\tilde{U}_{11}^{-1} h_{s}\right)^{n_{s}} b_{\mathrm{in}}^{+}\left(\tilde{U}_{22}^{-1} k_{1}\right)^{m_{1}} \\
& \ldots b_{\mathrm{in}}^{+}\left(\tilde{U}_{22}^{-1} k_{t}\right)^{m_{t}} \Omega_{\mathrm{in}} \\
& m_{i}, p_{i} \text { positive integers, } i=1, \ldots, s ; j=1, \ldots, t . \\
& |x-y|<\varepsilon .
\end{aligned}
$$

It suffices to show that $y$ is in the closure of the range $R(\mathfrak{U})$, of $\mathfrak{U}(t)$ which is in turn the case if any vector

$$
z=a_{\mathrm{in}}^{+}\left(\tilde{U}_{11}^{-1} h_{1}\right)^{n_{1}} \ldots a_{\mathrm{in}}^{+}\left(\tilde{U}_{11}^{-1} h_{s}\right)^{n_{s}} b_{\mathrm{in}}^{+}\left(\tilde{U}_{22}^{-1} k_{1}\right)^{m_{1}} \ldots b_{\mathrm{in}}^{+}\left(\tilde{U}_{22}^{-1} k_{t}\right)^{m_{t}} \Omega_{\mathrm{in}}^{\prime}
$$

is contained in $R(\mathfrak{H})$. To simplify the algebra we consider the case $m_{i}=0, i=1 \ldots t$, and make use of the identity on $\mathfrak{E}_{\mathrm{in}}$

$$
a_{\mathrm{in}}^{+}\left(\tilde{U}_{11}^{-1} h_{l}\right)=a_{l}^{\prime+}+\bar{\mu}_{l} b_{l}^{\prime} .
$$

Now $z$ can be rewritten

$$
z=(\chi \chi)^{-1 / 2} \prod_{l=1}^{s}\left(a_{l}^{\prime+}+\bar{\mu}_{l} b_{l}^{\prime}\right)^{n_{l}} \exp -\sum_{i} \mu_{i} a_{i}^{+} b_{i}^{\prime+} \Omega_{t}
$$

and is therefore (Section 3, Remark (4.18)) the strong limit of the sequence $\left\{z_{N}\right\}$

$$
z_{N}=(\chi \chi)^{-1 / 2} \prod_{l=1}^{s}\left(a_{l}^{\prime+}+\bar{\mu}_{l} b_{l}^{\prime}\right)^{n_{l}} \prod_{i=1}^{N} e^{-\mu_{l} a_{l}^{\prime}+b_{l}^{\prime+}} \Omega_{t} .
$$

$z_{N}$ is the limit of the sequence $\left\{z_{N M}\right\}$

$$
z_{N M}=(\chi \chi)^{-1 / 2} \prod_{l=1}^{s}\left(a_{l}^{\prime+}+\bar{\mu}_{l} b_{l}^{\prime}\right)^{n_{l}} \prod_{i=1}^{N}\left(\sum_{k=0}^{M}\left(k^{\prime}\right)^{-1}\left(-\mu_{i} a_{i}^{+} b_{i}^{+}\right)^{k}\right) \Omega_{t} .
$$

Hence the diagonal elements $w_{N}=z_{N N}$ are converging towards $z$. But $w_{N}$ is by construction an element in the range of $\mathfrak{U}(t)$.

According to Theorem 3 it is sufficient for existence and uniqueness (up to a phase) of the time evolution operator $\mathfrak{U}(t)$ and in particular of the 
$S$-matrix to investigate the operator $L(t)$ and to prove $L(t)$ to be H.S. That this is indeed the case we will quote without proof [7]:

Theorem 4. Let $\tilde{U}$ be the operator of the corollary to Theorem 1. Then the operator

$$
L(t)=\tilde{U}_{11}^{-1}(t) \tilde{U}_{12}(t)
$$

is Hilbert-Schmidt.

Remark. Due to the fact that $\tilde{U}_{11}$ and $\tilde{U}_{11}^{-1}$ is bounded the condition in Theorem 4 can be replaced by

$$
\left\|\tilde{U}_{12}(t)\right\|_{H S}<\infty .
$$

\section{Quantum Theory for Spin $1 / 2$ Particles}

As in the previous case the solution of the $c$-number Cauchy problem can be used to generate an automorphism $\alpha_{t}$ on the $C^{*}$-algebra $\mathfrak{Q}_{\text {in }}\left(\mathfrak{S}_{1 / 2}\right)$ generated by creation and annihilation operators of the in-field,

$$
\phi_{\text {in }}(f)=\left(\begin{array}{c}
a\left(f_{1}\right) \\
b^{+}\left(f_{2}\right)
\end{array}\right) \quad f=\left(\begin{array}{l}
f_{1} \\
f_{2}
\end{array}\right) \in \mathfrak{H}_{1 / 2} .
$$

$\mathfrak{Q}_{\text {in }}\left(\mathfrak{H}_{1 / 2}\right)$ is isomorphic to a selfdual canonical anticommutation relation $(S D C)$ algebra $\mathfrak{Q}_{S D C}(\mathfrak{\Re}, \Gamma)[8]$ defined as follows: Let $\Re$ be a complex Hilbert-space

$$
\begin{aligned}
\Re & =\left(L^{2}\left(d^{3} p\right) \otimes C^{2}\right) \otimes\left(L^{2}\left(d^{3} p\right) \otimes C^{2}\right) \otimes\left(L^{2}\left(d^{3} p\right) \otimes C^{2}\right) \otimes\left(L^{2}\left(d^{3} p\right) \otimes C^{2}\right) \\
F & =\left(\begin{array}{l}
f_{1} \\
f_{2} \\
f_{3} \\
f_{4}
\end{array}\right) \\
(F, F) & =\sum_{l=1}^{4} \sum_{s=1}^{2} \int d^{3} p\left|f_{l}(p, s)\right|^{2}
\end{aligned}
$$

and $\Gamma$ the antiunitary involution

$$
\Gamma F=\left(\begin{array}{l}
\bar{f}_{3} \\
\overline{f_{4}} \\
\bar{f}_{1} \\
\bar{f}_{2}
\end{array}\right) .
$$


The algebra $\mathfrak{Q}_{S D C}(\mathfrak{R}, \Gamma)$ is generated by operators $B(F)$ given in terms of a free spin $1 / 2$ field

$$
\begin{aligned}
\psi(x) & =\left(\frac{1}{2 \pi}\right)^{3 / 2} \int d^{3} p\left(\frac{m}{\omega(p)}\right)^{1 / 2}\left[e^{-i p x} u(p, s) a(p, s)+e^{i p x} v(p, s) b^{+}(p, s)\right] \\
B(F) & =\int d^{3} p f_{1}(p, s) a(p, s)+\int d^{3} p f_{2}(p, s) b^{+}(p, s) \\
& +\int d^{3} p f_{3}(p, s) a^{+}(p, s)+\int d^{3} p f_{4}(p, s) b(p, s) .
\end{aligned}
$$

$B$ and it's adjoint have the following properties characteristic for generators of an abstract $S D C$ algebra:

i) $B(F)$ is linear in $F$.

ii) $\left\{B(F) B^{+}(G)\right\}=(G, F)$.

iii) $B^{+}(F)=B(\Gamma F)$.

There is a subspace of $\mathfrak{A}$ isomorphic to $\mathfrak{H}_{1 / 2}$ with the projection operator $P$

$$
P F=\left(\begin{array}{l}
0 \\
f_{2} \\
f_{3} \\
0
\end{array}\right) .
$$

One checks readily that

$$
\Gamma P \Gamma+P=1
$$

which is the defining property of a basis projection [8].

Having defined $B$ in terms of $\psi$ one can consider $B$ in the Fock representation of $\psi$ with vacuum $\Omega$, and calculate the two point function, it turns out that

$$
\left(\Omega B^{+}(F) B(G) \Omega\right)=(F, P G) .
$$

In particular

$$
\left(\Omega, B(F) B^{+}(G) \Omega\right)=0 \quad \forall G \in P \Omega .
$$

The last equation is the defining property of the Fock state on $\mathfrak{Q}_{S D C}(\mathfrak{\Re}, \Gamma)$ in the terminology of Araki.

The $q$-number Cauchy problem is now solved using the results of the corresponding $c$-number problem as in the previous case (Theorem 2 ).

Theorem 5. If $A_{\mu}(x)$ are test functions, $A_{\mu} \in f\left(R^{4}\right)$, and $A_{\mu}(t, \boldsymbol{x})=0$ for $|t|>T$ the mapping $\alpha_{t}$ defined by

$$
\begin{aligned}
\alpha_{t} \phi_{\mathrm{in}}(\boldsymbol{x}) & =\left(\begin{array}{c}
a_{t}(\boldsymbol{x}, s) \\
b_{t}^{+}(\boldsymbol{x}, s)
\end{array}\right)=\tilde{U}(t) \phi_{\mathrm{in}}(\boldsymbol{x}) \\
\tilde{U}(t) & :=\tilde{U}(t,-T)
\end{aligned}
$$


generates an automorphism of $\mathfrak{Q}_{\mathrm{in}}\left(\mathfrak{S}_{1 / 2}\right)$ with the properties

i) $\alpha_{t} \phi_{\text {in }}(\boldsymbol{x})$ is the unique solution of the q-number Cauchy problem (1.2).

ii) $\alpha_{t}$ is local.

iii) $\alpha_{t}$ is a Bogoliubov automorphism.

Proof. $1 . \alpha_{t}$ is an automorphism of $\mathfrak{Q}_{\text {in }}\left(\mathfrak{S}_{1 / 2}\right)$. This is an immediate consequence of the well known relation (notation (5.1))

$$
\left\|a_{i \mathrm{i}}^{\sharp}(f)\right\| \leqq(f, f),\left\|b_{\text {in }}^{\sharp}(g)\right\| \leqq(g, g)
$$

and of Lemma 3, setting forth among other statements that $\tilde{U}$ mapps $\mathfrak{S}_{1 / 2}$ onto $\mathfrak{H}_{1 / 2}$.

2. $\alpha_{t} \phi_{\text {in }}(x)$ is a solution of the $q$-number Cauchy problem. Due to (5.10) and the second statement of Lemma 3 one gets

$$
\begin{aligned}
i \partial_{t}\left(\alpha_{t} \phi_{\mathrm{in}}\right)(f) & =\phi_{\mathrm{in}}\left(i \partial_{t} \tilde{U}^{T}(t) f\right) \\
& =\phi_{\mathrm{in}}\left(H_{1}^{T}(t) \tilde{U}(t) f\right)
\end{aligned}
$$

where $\tilde{U}^{T}$ denotes the transposed operator of $\tilde{U}$. It remains to check the initial data, however this is straightforward.

3 . The proof of uniqueness and locality parallels the corresponding statement for the case of spin zero particles and will not be given here. We are left with demonstrating the last statement of Theorem $5, \alpha_{t}$ is a Bogoliubov automorphism,

$$
\begin{aligned}
& \left\{a_{t}(f), a_{t}^{+}(g)\right\}=(f, g) \quad f \in L^{2} \otimes C^{2}, g \in L^{2} \otimes C^{2} \\
& \left\{b_{t}(f), b_{t}^{+}(g)\right\}=(f, g) .
\end{aligned}
$$

Concentrating on the first equation we get due to (5.9) for the left hand side

$$
\begin{aligned}
\left.\left\{a_{\mathrm{in}}\left(\tilde{U}_{11}^{+} f\right)=b_{\mathrm{in}}^{+} \overline{\left(\tilde{U}_{12}^{+} f\right.}\right), a_{\mathrm{in}}^{+}\left(\tilde{U}_{11}^{+} g\right)+b_{\mathrm{in}} \overline{\left(\tilde{U}_{12}^{+} g\right)}\right\} & =\left(f,\left(\tilde{U}_{11} \tilde{U}_{11}^{+}+\tilde{U}_{12} \tilde{U}_{12}^{+}\right) g\right) \\
& =(f, g) .
\end{aligned}
$$

The last step makes use of the canonical structure of the free spin $1 / 2$ field and unitary of $\tilde{U}$ (Lemma 3). All the other commutators are computed the same way.

Now we have to discuss whether the automorphism $\alpha_{t}$ of $\mathfrak{Q}_{\text {in }}\left(\mathfrak{H}_{1 / 2}\right)$ can be unitarily implemented in the Fock space $\mathfrak{F}_{\text {in }}$ of the in-field. According to a theorem of Shale and Stinespring $[6,8]$ it is sufficient for a unitary operator $\mathfrak{U}(t)$

$$
\begin{aligned}
& a_{t}(f)=\mathfrak{U}(t) a_{\text {in }}(f) \mathfrak{U}^{-1}(t) \\
& b_{t}(f)=\mathfrak{U}(t) b_{\text {in }}(f) \mathfrak{U}^{-1}(t)
\end{aligned}
$$

to exist, that $\tilde{U}_{12}$ is a Hilbert-Schmidt operator in $\mathfrak{Y}_{1 / 2} \cdot \mathfrak{U}(t)$ is unique up to a phase due to the irreducibility of the Fock representation of 
$\mathfrak{Q}_{\text {in }}\left(\mathfrak{H}_{1 / 2}\right)$. However we are going to impose some more stringent assumptions on $\tilde{U}$ in order to get a closed formula for the vacuum state $\Omega_{t}$ of $a_{t}$ and $b_{t}$ analogous to (4.11). One can not expect the condition $\left\|\tilde{U}_{12}\right\|_{H S}<\infty$ to be sufficient for $\Omega_{t}$ to be of the form (4.11) as the following arguments shows.

Let $a$ and $b$ be two fermion operators and $\Omega$ their vacuum

$$
\begin{aligned}
a \Omega & =b \Omega=0 \\
\left\{a, a^{+}\right\} & =\left\{b, b^{+}\right\}=1 .
\end{aligned}
$$

The Bogoliubov transformation

$$
\begin{aligned}
a_{\beta} & =a \cos \beta+b^{+} \sin \beta \\
b_{\beta}^{+} & =-a \sin \beta+b^{+} \cos \beta
\end{aligned}
$$

can be implemented by the unitary transformation

$$
\begin{aligned}
U(\beta) & =\exp -\beta\left(a b-b^{+} a^{+}\right) \\
a_{\beta} & =U(\beta) a U^{-1}(\beta) \\
b_{\beta} & =U(\beta) b U^{-1}(\beta) .
\end{aligned}
$$

The vacuum $\Omega_{\beta}$ can be computed and turns out to be

$$
\Omega_{\beta}=\left(1+\operatorname{tg}^{2} \beta\right)^{-1 / 2} e^{-\operatorname{tg} \beta a^{+} b^{+}} \Omega
$$

which makes sense only for $|\operatorname{tg} \beta| \neq \infty$. Notice that (5.18) is a simple expression in terms of $\operatorname{tg} \beta$ but not in $\beta$. It is this reason that makes the formula superior to

$$
\Omega_{\beta}=e^{-\beta\left(a b-b^{+} a^{+}\right)} \Omega .
$$

The following theorem contains the generalization of (5.18) to the case of a field.

Theorem 6. Let $\tilde{U}$ be a unitary operator in $\mathfrak{H}_{1 / 2}=L^{2}\left(d^{3} x\right) \otimes C^{4}$ and $\varepsilon>0$ such that

$$
\begin{aligned}
& \left\|\tilde{U}_{11}-1\right\| \leqq 1-\varepsilon \\
& \left\|\tilde{U}_{12}\right\|_{H S}<\varepsilon
\end{aligned}
$$

where $\|\cdot\|\left(\|\cdot\|_{H S}\right)$ denotes the operator (Hilbert-Schmidt) norm in $L^{2}\left(d^{3} x\right) \otimes C^{2}$. Then the operator

$$
L=\tilde{U}_{11}^{-1} \tilde{U}_{12}
$$

is a well defined H.S. operator,

$$
\begin{aligned}
L= & \sum \lambda_{i} f_{i} \otimes g_{i}, \Sigma \lambda_{i}^{2}<\infty 1>\lambda_{i} \geqq 0 \\
& \left\{f_{i}\right\},\left\{g_{i}\right\} \text { each orthogonal basis in } L^{2}\left(d^{3} x\right) \otimes C^{2}
\end{aligned}
$$


and the nontrivial vector $\chi_{t}$

$$
\chi_{t}=\exp -\sum_{i} \lambda_{i} a_{i}^{+} b_{i}^{+} \Omega_{\mathrm{in}}, \quad a_{i}=a_{\mathrm{in}}\left(f_{i}\right), \quad b_{i}=b_{\mathrm{in}}\left(g_{i}\right)
$$

is up to normalization a vacuum $\Omega_{t}$ for $\alpha_{t} \phi_{\text {in }}$

$$
\Omega_{t}=\left(\chi_{t} \chi_{t}\right)^{-1 / 2} \chi_{t} \text {. }
$$

$\Omega_{t}$ is unique up to a phase.

Remark. Proceeding as in the case of spin zero one could make use of the explicit form of $\Omega_{t}$ and construct the unitary time evolution operator $\mathfrak{U}(t)$.

Proof $1 . L$ is a well defined H.S. operator and $0 \leqq \lambda_{i}<1: \tilde{U}_{11}$ has a bounded inverse

$$
\left\|\tilde{U}_{11}^{-1}\right\| \leqq \frac{1}{\varepsilon}
$$

and therefore

$$
\left\|\tilde{U}_{11}^{-1} \tilde{U}_{12}\right\|_{H S} \leqq\left\|\tilde{U}_{11}^{-1}\right\|\left\|\tilde{U}_{12}\right\|_{H S}<1 .
$$

2. $\chi_{t}$ is well defined and nonvanishing: Consider the sequence $\left\{\chi_{N}\right\}$,

$$
\chi_{N}=\prod_{i=1}^{N} e^{-\lambda_{i} a_{i}^{+} b_{i}^{+}} \Omega_{\text {in }} .
$$

In analogy to the spin zero case we claim that $\left\{\chi_{N}\right\}$ is a Cauchy sequence. A straightforward calculation yields:

$$
\left\|\chi_{N}-\chi_{M}\right\|^{2}=\left|\prod_{i=1}^{M}\left(1+\lambda_{i}^{2}\right)-\prod_{i=1}^{N}\left(1+\lambda_{i}^{2}\right)\right| .
$$

However the infinite product

$$
\prod_{i=1}^{\infty}\left(1+\lambda_{i}^{2}\right)
$$

converges if and only if $\Sigma \lambda_{i}^{2}<\infty$ [17].

3. $\chi_{t}$ is annihilated by $a_{t}$ and $b_{t}$ : We concentrate on the second of the two relations:

$$
\begin{aligned}
& a_{t}(f) \chi_{t}=\left(a_{\mathrm{in}}\left(\tilde{U}_{11}^{+} f\right)+b_{\mathrm{in}}^{+}\left(\tilde{U}_{12}^{+} f\right)\right) \exp -\Sigma \lambda_{i} a_{i}^{+} b_{i}^{+} \Omega_{\mathrm{in}}=0, \\
& b_{t}(f) \chi_{t}=\left(a_{\mathrm{in}}^{+}\left(\tilde{U}_{21}^{+} \bar{f}\right)+b_{\mathrm{in}}\left(\tilde{U}_{12}^{+} f\right)\right) \exp -\Sigma \lambda_{i} a_{i}^{+} b_{i}^{+} \Omega_{\mathrm{in}}=0 .
\end{aligned}
$$

Calculating formally only because the necessary justifications are simple to provide due to the last paragraph, we compute first

$$
\left[b\left(\tilde{U}_{22}^{T} f\right), e^{-\lambda_{i} a_{i}^{+} b_{i}^{+}}\right]=\lambda_{i} a_{i}^{+}\left(g_{i}, \tilde{U}_{22}^{+} \bar{f}\right) e^{-\lambda_{1} a_{i}^{+} b_{i}^{+}}
$$


which leads to

$$
\left[b\left(\tilde{U}_{22}^{T} f\right), \exp -\sum_{i} a_{i}^{+} b_{i}^{+}\right]=\left(\exp -\sum_{i} a_{i}^{+} b_{i}^{+}\right) a^{+}\left(L \tilde{U}_{22}^{+} \bar{f}\right) .
$$

The second factor on the right hand side can be computed using the unitarity relation

$$
\tilde{U}_{11} \tilde{U}_{21}^{+}+\tilde{U}_{12} \tilde{U}_{22}^{+}=0
$$

and yields

$$
a^{+}\left(L \tilde{U}_{22}^{+} \bar{f}\right)=-a^{+}\left(\tilde{U}_{21} \bar{f}\right)
$$

proving (5.29).

4. Uniqueness of the vacuum can be derived either by applying the same method as in the previous case or making use of the theorem by Shale and Stinespring [6].

The remaining part of this section will be an application of Theorem 6 to the automorphisme $\alpha_{t}$ of $\mathfrak{Q}_{\text {in }}\left(\mathfrak{H}_{1 / 2}\right)$ defined by (5.9). As a first step we are going to compare the assumptions made in theorem 6 with the ones in the theorem of Shale and Stinespring (formulation of Araki) as anticipated previously. The isometry of $\mathfrak{Q}_{S D C}(\mathfrak{R}, \Gamma)$ and $\mathfrak{Q}_{\text {in }}\left(\mathfrak{H}_{1 / 2}\right)$ induces in $\mathfrak{Q}_{S D C}(\mathfrak{\Re}, \Gamma)$ an automorphism corresponding to $\alpha_{t}$ again denoted by the same symbol. $\alpha_{t}$ is given explicitly by

$$
\begin{aligned}
\alpha_{t} B(F) & =B\left(F_{t}\right) \\
F_{t} & =\left(\begin{array}{cc}
\tilde{U}^{T}(t) & 0 \\
0 & \tilde{U}^{+}(t)
\end{array}\right)\left(\begin{array}{l}
f_{1} \\
f_{2} \\
f_{3} \\
f_{4}
\end{array}\right) .
\end{aligned}
$$

The necessary and sufficient condition for $\alpha_{t}$ to be unitarily implementable in the Fock representation of $\mathfrak{Q}_{S D C}(\Re, \Gamma)$ is [8]

$$
\left\|(1-P)\left(\begin{array}{cc}
\tilde{U}^{T}(t) & 0 \\
0 & \tilde{U}^{+}(t)
\end{array}\right) P\right\|_{H S}<\infty .
$$

One readily verifies that (5.35) is equivalent to

$$
\begin{gathered}
\left\|\tilde{U}_{12}\right\|_{H S}<\infty \\
\left\|\tilde{U}_{21}^{T}\right\|_{H S}<\infty \\
\tilde{U}(t)=\left(\begin{array}{ll}
\tilde{U}_{11} & \tilde{U}_{12} \\
\tilde{U}_{21} & \tilde{U}_{22}
\end{array}\right) .
\end{gathered}
$$


The first inequality is part of the assumption of Theorem 6 . The second one is satisfied provided $\tilde{U}_{11}^{-1}$ and $\tilde{U}_{22}^{+}$are bounded in norm, which they are (5.20), since

$$
\tilde{U}_{21}^{+}=\tilde{U}_{11}^{-1} \tilde{U}_{12} \tilde{U}_{22}^{+}
$$

due to the unitarity relations for $\tilde{U}$. Hence the assumptions of Theorem 6 imply (5.35).

Next we are going to demonstrate that under the conditions of Theorem 5 together with $\boldsymbol{A}=0$ the statement of Theorem 6 is applicable, i.e. there is a $t_{0}>-T$ such that for $t<t_{0}$ the assumptions of Theorem 6 (5.20) are satisfied. In fact it is only necessary to show the second inequality because $\tilde{U}(t)$ is norm continuous and $\tilde{U}(-T)=1$. The following result reduces the proof to a discussion of $\tilde{H}_{1}$ :

Lemma 4. Let $\tilde{U}(t)$ be the time evolution operator of the Cauchy problem $(3.8)$ and

$$
c(t):=1-2(t+T) \sup _{-T \leqq s \leqq t}\left\|\tilde{H}_{1}(s)\right\|>0 .
$$

Then

$$
\begin{gathered}
d(t):=\sup _{-T \leqq S \leqq t}\left\|\int_{-T}^{s} d t\left(\tilde{H}_{1}\right)_{12}\right\|_{H S} \\
\left\|\tilde{U}_{12}(t)\right\|_{H S}<c^{-1}(t) d(t) .
\end{gathered}
$$

Proof. As it has been remarked earlier (Section 3) $\tilde{U}(t)$ can be represented in terms of a norm converging Dyson series. The $n$-th term of the series contributes to $\tilde{U}_{12}$ be means of $2^{\mathrm{n}}$ multiple integrals of the form

$$
\begin{gathered}
A\left(i_{l}, h_{l}\right)=(-i)^{n} \int_{-T}^{t} d t_{1}\left(\tilde{H}_{1}\right)_{i_{1} k_{1}} \ldots \int_{-T}^{t_{k-1}} d t_{k}\left(\tilde{H}_{1}\right)_{12} \ldots \int_{-T}^{t_{n-1}} d t_{n}\left(\tilde{H}_{1}\right)_{i_{n} k_{n}} \\
i_{l}, k_{l}=1,2, \quad l=1 \ldots n .
\end{gathered}
$$

The H.S. norm of $A\left(i_{l}, k_{l}\right)$ can be estimated as follows

$$
\left\|A\left(i_{l}, k_{l}\right)\right\|_{H S} \leqq(t+T)^{n-1}\left(\sup _{-T \leqq s \leqq t}\left\|\tilde{H}_{1}(s)\right\|\right)^{n-1} d(t) .
$$

Hence

$$
\left.\left\|\tilde{U}_{12}(t)\right\|_{H S} \leqq 2 \sum_{n=1}^{\infty}\left[2(t+T)\left(\sup _{-T \leqq s \leqq t}\left\|\tilde{H}_{1}(s)\right\|\right)\right]\right]^{n-1} d(t) .
$$

For $t$ being sufficiently close to $-T(5.38)$ the sum converges and yields

$$
\left\|\tilde{U}_{12}(t)\right\|_{H S} \leqq \frac{2 d(t)}{1-2(t+T) \sup _{-T \leqq s \leqq t}\left\|\tilde{H}_{1}(s)\right\|} .
$$


Notice that $c(t)$ obeys a Lipschitz condition at $t=-T$ due to it's definition

$$
|c(t)-1| \leqq \operatorname{const}(t+T), \quad t \geqq-T .
$$

For the rest of the proof it is therefore sufficient to demonstrate the continuity of $d(t)$ at $t=-T$.

Lemma 5. For any $\varepsilon>0$ there exists a $t_{0}>-T$ such that

$$
d(t)<\varepsilon \quad V-T \leqq t \leqq t_{0}
$$

provided the external field is merely electric, $\boldsymbol{A}=0$.

In accordance with the previous definition (3.8) the operator $\tilde{H}_{1}$ has the following representation in momentum space

$$
\begin{aligned}
\tilde{H}_{1}(q, p)= & \left(\frac{m}{\omega(p)}\right)^{1 / 2}\left(\begin{array}{ll}
e^{i \omega(q) t} & 0 \\
0 & e^{-i \omega(q) t}
\end{array}\right) \\
& \cdot\left(\begin{array}{ll}
\tilde{u}(-q, s) A(q-p) u(-p, r) & \tilde{u}(-q, s) A(q-p) v(+p, r) \\
\tilde{v}(+q, s) A(q-p) u(-p, r) & \tilde{v}(+q, s) A(q-p) v(+p, r)
\end{array}\right) \\
& \cdot\left(\begin{array}{ll}
e^{-i \omega(q) t} & 0 \\
0 & e^{i \omega(q) t}
\end{array}\right)\left(\frac{m}{\omega(q)}\right)^{1 / 2} .
\end{aligned}
$$

A straightforward calculation using the relations

yields

$$
\begin{aligned}
\sum_{s} u(p, s) \tilde{u}(p, s) & =\frac{\not p+m}{2 m} \\
-\sum_{s} v(p, s) \tilde{v}(p, s) & =\frac{-p+m}{2 m}
\end{aligned}
$$

$$
\begin{aligned}
\left\|\int_{-T}^{s} d t\left(\tilde{H}_{1}\right)_{12}\right\|_{H S}^{2}= & -m^{2} \int \frac{d^{3} p}{\omega(p)} \frac{d^{3} q}{\omega(q)} \int_{-\boldsymbol{T}}^{s} d t d t^{\prime} \operatorname{Trace} A(\boldsymbol{q}-\boldsymbol{p}) \\
& \cdot \gamma^{0}\left(\frac{\phi-m}{2 m}\right) \gamma^{0} A(\boldsymbol{q}-\boldsymbol{p})\left(\frac{\not p+m}{2 m}\right) e^{-i(\omega(q)+\omega(p))\left(t-t^{\prime}\right)} .
\end{aligned}
$$

The trace can be easily computed using well known rules,

$$
\begin{aligned}
\left\|\int_{-T}^{s} d t\left(\tilde{H}_{1}\right)_{12}\right\|_{H S}^{2}= & \int \frac{d^{3} p}{\omega(p)} \frac{d^{3} p}{\omega(q)} \int_{-T}^{s} d t d t^{\prime}\left[2 \operatorname{Re}(A p)(\bar{A} q)_{e}\right. \\
& \left.-(\bar{A} A)(p, q)_{e}-m^{2}(\bar{A} A)\right] e^{-i(\omega(q)+\omega(p))\left(t-t^{\prime}\right)}
\end{aligned}
$$

where $(,)_{e}$ denotes the Euclidian sclar product. A power counting argument shows that the H.S. norm can not expected to be finite for 
arbitrary external fields. For an electric field only (5.47) specializes to

$$
\begin{aligned}
\left\|\int_{-T}^{s} d t\left(\tilde{H}_{1}\right)_{12}\right\|_{H S}^{2}= & \int \frac{d^{3} p}{\omega(p)} \frac{d^{3} q}{\omega(q)} \int_{-T}^{s} d t d t^{\prime} \bar{A}_{0}(t, \boldsymbol{p}-\boldsymbol{q}) A_{0}\left(t^{\prime}, \boldsymbol{p}-\boldsymbol{q}\right) \\
& \cdot\left((p q)-m^{2}\right) e^{-i(\omega(q)+\omega(p))\left(t-t^{\prime}\right)} .
\end{aligned}
$$

A partial integration in $t$ and $t^{\prime}$ leads to

$$
\begin{aligned}
\left\|\int_{0}^{s} d t\left(\tilde{H}_{1}\right)_{12}\right\|_{H S}^{2}= & \int d^{3} p d^{3} q I_{1}(p, q) J(s, p, q) \\
I_{1}(p, q):= & \frac{p q-m^{2}}{\omega(p) \omega(q)(\omega(p)+\omega(q))^{2}} \\
J(s, \boldsymbol{p}, \boldsymbol{q}):= & |\alpha(s)|^{2}+\int_{0}^{s} d t d t^{\prime} \alpha(t) \alpha\left(t^{\prime}\right) e^{-i(\omega(p)+\omega(q))\left(t-t^{\prime}\right)} \\
& +2 \operatorname{Re} \alpha(s) e^{-i(\omega(p)+\omega(q)) s} \int_{0}^{s} d t \alpha(t) e^{i(\omega(p)+\omega(q) t} \\
\alpha(s):= & \int_{0}^{s} d t A_{0}(t, \cdot)
\end{aligned}
$$

where we set $T=0$ for convenience. Since $I_{1}(p, q)$ is bounded and $J(s, p, q)$ converges pointwise to zero for $s \rightarrow 0$ it is sufficient to find an integrable function majorizing $I_{1}(p, q)$. For $J$ we use the crude estimate

$$
J(s, \boldsymbol{p}, \boldsymbol{q}) \leqq \text { const. } \sup _{0 \leqq t \leqq s}|A(t, \boldsymbol{p}-\boldsymbol{q})|^{2} .
$$

It is therefore sufficient to discuss the integral

$\int d^{3} p d^{3} q|A(t, \boldsymbol{p}-\boldsymbol{q})|^{2} I_{1}(p, q)=\int d^{3} r|A(t, r)|^{2} \int d^{3} p I_{1}(p,|\boldsymbol{p}-\boldsymbol{r}|)$.

$I_{1}(p,|\boldsymbol{p}-\boldsymbol{r}|)$ is smaller than $I_{2}(\boldsymbol{p}, \boldsymbol{r})$

$$
I_{2}(\boldsymbol{p}, \boldsymbol{r}):=\frac{\omega(p) \omega(p-r)-\boldsymbol{p} \boldsymbol{r}-\omega^{2}(p)}{\sqrt{p^{2}+m^{2}} \sqrt{(p-r)^{2}+m^{2}}\left(\sqrt{p^{2}+m^{2}}+\sqrt{(p-r)^{2}+m^{2}}\right)^{2}} .
$$

Integrating over angles $d \Omega(p)$ results in

$$
\begin{aligned}
& \frac{1}{4 \pi} \int d \Omega(p) I_{2}(\boldsymbol{p}, \boldsymbol{r}) \\
& \quad=\frac{(6 p r)^{-1}\left[\left(m^{2}+(p+r)^{2}\right)^{3 / 2}-\left(m^{2}+(p-r)^{2}\right)^{3 / 2}\right]-\sqrt{m^{2}+p^{2}}}{\sqrt{m^{2}+(p-r)^{2}}\left(\sqrt{m^{2}+p^{2}}+\sqrt{m^{2}+(p-r)^{2}}\right)^{2}} .
\end{aligned}
$$

One readily verifies that the above expression is integrable in $p$ because in the numerator the dominant parts cancel. A straightforward analysis 
leads to

$$
\int d^{3} p I_{2}(p, r) \leqq \text { const }(r+1) \text {. }
$$

Hence

$$
\left(\sup _{0 \leqq t \leqq s}|A(t, \boldsymbol{p}-\boldsymbol{q})|\right) I_{2}(\boldsymbol{p}, \boldsymbol{q})
$$

is integrable and majorizes by construction the integrand $I_{1} J$. Hence we proved the following result:

Theorem 7. Provided the assumptions of Theorem 5 are met and $\boldsymbol{A}=0$, there exists a constant $t_{0}>-T$ such that inequalities (5.20) are satisfied for $-T \leqq t \leqq t_{0}$.

Finally we are going to indicate how the previous argument has to be altered such as to give a proof of existence of a unitary $S$-matrix under the assumption of Theorem 5. For $t_{0}>t$ the $S$-matrix can be constructed as in the case of spin zero (4.25) as has been shown earlier. In order to construct the $S$-matrix by a finite number of similar steps one has to prove that the lengths of the intervals on the time axis can be chosen uniformly in length. The key to this statement is the following strengthened version of Lemma 5:

Lemma 6. Provided the external field is purely electric, $\boldsymbol{A}=0, d(t)$ vanishes at $t=-T$ as $(t+T)$ :

$$
d(t)=\text { const }(t+T) .
$$

The proof of Lemma 6 follows the one of Lemma 5 up to inequality (5.5) which is replaced by $(T=0)$

$$
J(s, \boldsymbol{p}, \boldsymbol{q}) \leqq s^{2} \text { const } \sup _{0 \leqq t \leqq s}|A(t, \boldsymbol{p}-\boldsymbol{q})|^{2}+\left(\sup _{0 \leqq t \leqq s}\left|\operatorname{grad}_{p} A(t, \boldsymbol{p}-\boldsymbol{q})\right|^{2}\right) \text {. }
$$

Hence

$$
\left\|\int_{0}^{s} d t\left(\tilde{H}_{1}\right)_{12}\right\|_{H S}^{2} \leqq s^{2} \chi^{2} \sup _{0 \leqq t \leqq s}\|A(t, \cdot)\|_{1}^{2}
$$

where \|\|$_{1}$ denotes the Sobolev norm $\|\cdot\|_{1}^{2}=\|\cdot\|^{2}+\|\operatorname{grad} \cdot\|^{2}$ and \|\| the usual $L_{2}$-norm. The constant $\chi$ is independent of $A$ and $s$. Combining (5.56) and (5.42) leads to

$$
\left\|\tilde{U}_{12}(t)\right\|_{H S} \leqq \frac{2 \chi \sup _{-T \leqq s \leqq t}\|A(s, \cdot)\|_{1}}{1-2(t+T) \sup _{-T \leqq s \leqq t}\left\|\tilde{H}_{1}(s)\right\|}(t+T)
$$

which shows that indeed the lengths of the intervals covering the set $(-T,+T)$ can be chosen uniformly. 
Remark: In $x$-space Eq. (5.46) reads

$$
\begin{aligned}
\left\|\int_{-T}^{s} d t\left(\tilde{H}_{1}\right)_{12}\right\|_{H S}^{2}= & -m^{2} \int d^{3} x d^{3} y \operatorname{Trace} A(x) \gamma^{0}\left(\frac{i \partial_{x}-m}{2 m} \Delta_{+}(x-y)\right) \\
& \cdot \gamma^{0} A(y)\left(\frac{i \partial_{y}+m}{2 m} \Delta_{+}(x-y)\right) .
\end{aligned}
$$

\section{References}

1. Feynman, R.P.: Phys. Rev. 76, 749 (1949).

2. Salam, A., Matthews, P.T.: Phys. Rev. 90, 690 (1953).

3. Schwinger, J.: Phys. Rev. 93, 615 (1954).

4. Capri, A.Z.: J. Math. Phys. 10, 575 (1969).

5. Boongarts, J. P. H.: Annals of Physics 56, 108 (1970).

6. Shale, D.: Trans. Am. Math. Soc. 103, 149 (1961).

- Stinespring, W.F.: J. Math. Mech. 14, 315 (1965).

7. Schroer, B., Seiler, R., Swieca, A.: Phys. Rev. D 2, 2932 (1970).

8. Araki,H.: On quasi-free states of CAR and Bogoliubov automorphisms, Preprint, Research Institute for Mathematical Sciences, Kyoto University, Kyoto, Japan, 1970.

9. Kristensen, P., Mejlbo, L., Thue Poulsen, E.: Commun. math. Phys. 6, 29 (1967).

10. Friedrichs, K., Lewy, H.: Math. Ann. 98, 192 (1928); see also K. Yosida [11].

11. Yosida, K.: Functional analysis. New York: Academic Press 1965.

12. Nelson, E., Flows: Preliminary informal notes. Princeton: University Press 1969, page 16.

13. Schweber, S.S.: Introduction to relativistic quantum field theory, p. 82. Evanston, IIl.: Row, Peterson and Company 1961.

14. Araki,H., Woods, E. J.: J. Math. Phys. 4, 637 (1963), lemma 2.3.

15. See e.g. Hepp, K.: Théorie de la rénormalization; Lecture Notes in Physics 2, p. 11. Berlin-Heidelberg-New York: Springer 1969.

16. Neumann, J.von: Comp. Math. 6, 1 (1938).

R. Seiler's present address

C.N.R.S.

Centre de Physique théorique

31 Chemin J. Aiguier

F-13 Marseille 9, France 